\title{
Interplanetary Disturbances Affecting Space Weather
}

\author{
Robert F. Wimmer-Schweingruber ${ }^{1}$ \\ ${ }^{1}$ Institute for Experimental and Applied Physics \\ Christian-Albrechts-University Kiel, \\ Kiel, Germany \\ email: wimmer@physik.uni-kiel.de
}

\begin{abstract}
The Sun somehow accelerates the solar wind, an incessant stream of plasma originating in coronal holes and some, as yet unidentified, regions. Occasionally, coronal, and possibly sub-photospheric structures, conspire to energize a spectacular eruption from the Sun which we call a coronal mass ejection (CME). These can leave the Sun at very high speeds and travel through the interplanetary medium, resulting in a large-scale disturbance of the ambient background plasma. These interplanetary CMEs (ICMEs) can drive shocks which in turn accelerate particles, but also have a distinct intrinsic magnetic structure which is capable of disturbing the Earth's magnetic field and causing significant geomagnetic effects. They also affect other planets, so they can and do contribute to space weather throughout the heliosphere. This paper presents a historical review of early space weather studies, a modern-day example, and discusses space weather throughout the heliosphere.
\end{abstract}

\section{Introduction}

On the tenth of September, 1580, the people of the town of Augsburg in Southern Germany saw "a large and very frightening magic sign in the sky after sunset" (Fig. 1). Its origins were unknown and even less understood, and hence the text in Fig. 1 continues in a religious manner. This heavenly appearance is likely to have been an aurora, a phenomenon usually not seen this far South in Europe. However, they were already known to the Romans, who termed them 'aurora', i.e., dawn, or the reddening of the morning sky. The description of the aurora in Fig. 1 is a witness that people were frightened by this unusual phenomenon which would take more than 300 years to be understood.

William Gilbert is traditionally credited with having initiated scientific studies of magnetism, especially the Earth's magnetism (Gilbert 1600). He correctly interpreted the deflection of a compass's needle as due to global terrestrial magnetism, stating that "Magnus magnes ipse est globus terrestris" (The Earth itself is a giant magnet). This then was an inadvertent first step towards understanding the origin of aurorae in a scientific way. Another ingredient is sunspots which as naked-eye phenomena were probably a puzzle already much earlier in history. For instance, Anaxagoras, a Greek philosopher, may have observed a sunspot in 467 BC (Bicknell 1968). Chinese astronomers reported sunspots in 164 B.C. (Clark \& Stephenson 1978, Wittmann \& Xu 1987), but we apparently have no records about Babylonian or Arab observations. It was Galileo who gave the correct interpretation despite the prevailing prejudice that sunspots must be transits of planets or moons (Galileo 2010). It is interesting to note that even in those times, priorities on discoveries were also bitterly disputed, in this case between Galileo and Scheiner (Galileo 2010). Sunspots were soon found to vanish nearly completely (during the so-called Maunder minimum (Eddy 1976)) and reappear around 1700. Schwabe (1844) showed clearly that the number of sunspots varies with a cycle (i.e., not an exact period) of roughly 


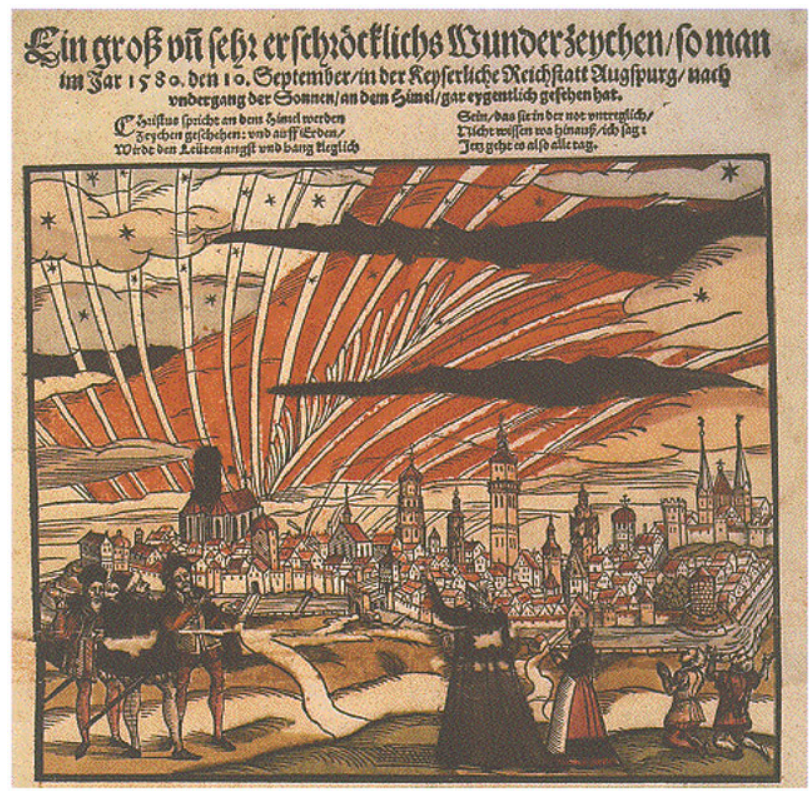

Figure 1. Medieval print showing aurorae at the southern German city of Augsburg in 1580. Reprinted with permission by Zentralbibliothek Zürich, Graphische Sammlung und Fotoarchiv.

11 years. Around this time, Carl Friederich Gauß together with his assistant, Wilhelm Weber, was studying the Earth's magnetic field and variations thereof. They set up the "Magnetic Group" ("Magnetischer Verein") and with the aid of Alexander von Humboldt set up a world-wide network of magnetometers (Stern 2002). This allowed them to monitor rapid changes in the Earth's magnetic field and ultimately led to the discovery of the magnetosphere, ionosphere, and disturbances in their normal state. One famous such disturbance was - in retrospect - caused by the famous Carrington flare. Carrington had reported that "... when ... two patches of intensely bright and white light broke out in the positions ... A and B, in the forms of the spaces left white." The account continues and mentions that "Mr Carrington ... pointed out ... that toward four hours after midnight there commenced a great magnetic storm,... While the contemporary occurrence may deserve noting, he would not have it supposed that he even leans toward hastily connecting them. 'One swallow does not make a summer."' (Carrington 1859). Kristian Birkeland, a Norwegian researcher who studied the aurora in two expeditions in 1902 and 1903 (Birkeland 1908 and 1913), showed that magnetic storms and aurorae were intimately linked (Birkeland 1908), and that the Sun, solar (cathode) rays, and solar discharges cause them (Birkeland 1911).

Studies of ionizing radiation were much en vogue in the early twentieth century after the discovery of X-rays (Röntgen 1895) which earned him the Nobel proze in 1901. The penetrating nature of radiation was studied by Becquerel in 1896 (1896a, b) and by the Curies (1898a, b) which earned the three the Nobel prize in $1903 \dagger$, notably in the absence of the Curies. One of the researchers of these days was Victor Hess who investigated the ionization of air using an electrometer at different altitudes. He excluded the Sun as the ionizing source and concluded, after the final of a series of balloon flights, that there is a source of radiation which increases with height and enters the atmosphere from above (Hess 1912). He was not alone in investigating what Millikan later termed

$\dagger$ see http://www.nobelprize.org/nobel_prizes/physics/laureates/1903/press.html 


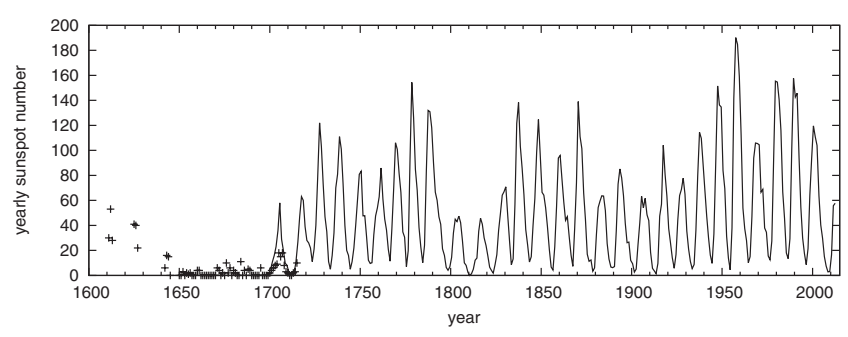

Figure 2. Yearly sunspot number plotted versus time. Early data are plotted as plus symbols, systematic data as a solid line. One easily recognizes the Maunder minimum in the second half of the seventeenth century. Data are from the SIDC-team (http://www.sidc.be/sunspot-data/).

'cosmic rays'. Pacini, an Italian researcher, performed similar experiments but also on the sea, on lakes, as well as under water. From these he also concluded that there must be radiation reaching the ground from above, i.e., through the atmosphere (Pacini 1912).

\subsection{Solar Activity}

The Sun is not constant, as can be seen in Fig. 2, which shows yearly sunspot numbers as obtained from http://www.sidc.be/sunspot-data/ as a solid line and earlier data as plus symbols. The well-known Maunder minimum is clearly seen in the second half of the seventeenth century, a period of time when the Sun was virtually spotless. Later, solar activity waxes and wanes on a semi-regular basis which is termed the solar cycle (Schwabe 1844). There are historical reconstructions of solar activity beyond the sunspot record (e.g., Eddy 1976) using cosmogenic isotopes, notably ${ }^{14} \mathrm{C}$ and ${ }^{10} \mathrm{Be}$ which are produced by interactions of galactic cosmic rays (GCR) with the Earth's atmosphere and can then be measured in terrestrial archives. Possible linkages with terrestrial climate, as first discussed at length, e.g., in Eddy (1976) (but see Usoskin 2013 for a more recent account), are certainly interesting, but will not be addressed here, as it is not the intent of the paper. Nevertheless, reconstructions of historical solar activity may allow us to better understand space weather today because only they can put the observations into a larger context. We must not forget that we have only just begun to understand space weather during the space age which itself is very short compared to the age of the Sun, the sunspot record, or longer solar activity cycles such as the $\sim 80$-year Gleissberg cycle (Wolf 1856, Gleissberg 1944, Eddy 1976). It is from such reconstructions that we understand that the Sun does not only vary with a 11 or 22-year cycle, but that there are longer cycles superimposed on this (e.g., Hathaway 2010), as can also be seen in Fig. 2. We can clearly see another minimum around 1800 (Dalton minimum) and another, less deep one around 1900. What is more conspicuous though, is the observation that the number of sunspots in the space age has been exceptionally high if viewed in a historical context. In fact, the sunspot number at maximum has been decreasing since the maximum in the late fifties, and there are indications that we may be leaving the 'Grand Solar Maximum' (e.g., Barnard et al. 2011) which has dominated the space age. This would entail an increase in the flux of cosmic rays, which indeed was observed during the last solar activity minimum in 2009/2010 when the intensities of heavy cosmic ray nuclei was $\sim 20 \%$ higher than in the previous minimum (Mewaldt et al. 2010).

I was asked to give a talk entitled "Interplanetary disturbances affecting space weather", and have just given a historical motivation for the study of space weather and some of the effects of space weather. It is now time to proceed with the actual topic - but what is it? What is a disturbance? What affects space weather? Where and what is 
space weather? This then is the plan of the paper: I will discuss the traditional aspects of space weather in the coming section (2), show that it matters at other locations than Earth in sections 3 and 3.2, and yes, even at other planets (section 3.3), and conclude in section 4 .

\section{What Affects Space Weather? - A Traditional Introduction}

So what is space weather and what affects it? As already alluded to above, geomagnetists knew about the solar influence on the Earth's magnetic state (e.g., Birkeland $1908,1911)$. More subtle variations than the spectacular aurorae were also uncovered to be induced by then Sun. Remarkably, even the virtually spotless Sun in the solar minimum around 1930 had a clear effect on the Earth's magnetism, as was recognized by Bartels (1934). The observation that there were steadily recurrent magnetic disturbances at Earth despite the fact that the Sun had hardly any spots (and thus was very inactive) had a profound implication. Looking at Figure 1 of Bartels (1934), one observes that the Sun has only few spots after about 1930 but that there is a strong pattern of recurrent geomagnetic disturbances which appeared to be stable for two or more years. Its frequency of recurrence was approximately once every 27 days, hinting at a link with the Sun and its 27-day rotation period. These recurrent magnetic disturbances were thought to be caused by so-called M-regions on the Sun (Bartels 1934) which remained, however, elusive, and could not be observed. Today we consider recurrent high-speed streams or rather their compressed leading edges (corotating interaction regions, CIRs) to be the origin of recurrent magnetic disturbances.

So it looks as if we have two ingredients of space weather at Earth, the leading edges of (not necessarily recurrent) high-speed streams or CMEs and/or particles from active regions on the Sun. Both can, but must not necessarily cause aurorae. Again, today we also understand the latter phenomenon a bit better. Active regions can somehow (we still disagree on how) be triggered (or trigger themselves) to rearrange magnetically, liberating a tremendous amount of energy in the form of radiation (photons and energetic particles) as well as (often, but not always) in the form of a coronal mass ejection (CME). Both can affect space weather, but in different ways. If you are interested in geomagnetic disturbances, then the CME is much more geoeffective than the energetic particles. If you are more interested in what will happen to astronauts in the International Space Station (ISS) or to electronics parts in satellites or other spacecraft, then the particles can be more important. So it looks very much as if space weather depends on your point of view! All three agents (high-speed streams (CIRs), flare particles, and CMEs) matter, but not at the same location and not for the same purpose.

CMEs or rather their interplanetary manifestations (ICMEs, see Wimmer-Schweingruber (2006), Wimmer-Schweingruber et al. (2006), Zurbuchen and Richardson (2006)) can strongly influence space weather. As CMEs leave the Sun, they carry with them an imprint of their solar origin in their magnetic structure (see, e.g., Crooker and Horbury 2006). Many ICMEs are magnetic clouds and can be recognized as a flux rope with a handedness (chirality) which is determined by Hale's law (Bothmer and Rust 1997). As they expand, their field becomes gradually more and more twisted in order to conserve both axial and azimuthal magnetic flux (e.g., Parker 1979). Thus, when a flux-rope ICME lies mainly in the ecliptic plane, it will have either at its leading or its trailing edge, a southward-pointing magnetic field component. A Southward-pointing leading edge, being compressed, is the more geoeffective because the Earth's magnetic field points roughly from the geographic South pole (approximately the magnetic North pole) to the geographic North pole (the magnetic South pole). Thus, the leading edge of the Earth's 


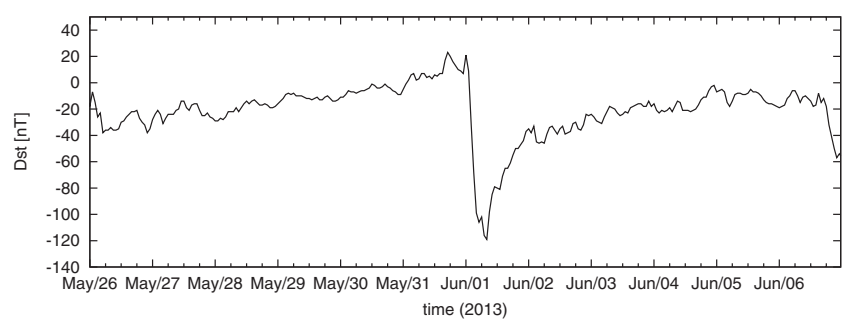

Figure 3. Major geomagnetic storm as observed on June 1, 2013. Data are from the Kyoto WDC (http://wdc.kugi.kyoto-u.ac.jp/wdc/Sec3.html).

magnetosphere has a northward-pointing magnetic field. As the southward interplanetary magnetic field (IMF) associated with an ICME hits the Earth's magnetic field, it can trigger magnetic reconnection (e.g., Parker 1979). In this case, the global field reconfigures itself, which induces a current, which in turn creates its own magnetic field. If the induced current returns in power lines or oil pipelines, this can adversely affect technological infrastructure. Today, such reconnection at the day side magnetosphere has been observed in situ, e.g., by the combined Cluster and Double Star missions (e.g., Dunlop et al. (2005)). Reconnection at the magnetotail and elsewhere can accelerate particles to sufficient energies that they can penetrate the uppermost layers of the Earth's atmosphere. After such a reconnection event, the polar regions are also open to the solar wind. Apart from the particles accelerated by the reconnection, the solar wind can als interact with upper atmospheric nitrogen and oxygen to form aurorae in the polar regions. The green and dark-reddish aurorae come from recombining oxygen, the blueish and light-red ones come from nitrogen.

The difficulty in predicting the geoeffectiveness of CMEs vs. CIRs was nicely illustrated in the week just before this workshop. A major geomagnetic storm with a Dst $<-100$ nT was observed at Earth on June 1, 2013, as shown in Fig. 3. What caused it? Initial inspections of images from the Solar Dynamics Observatory (SDO) revealed a pronounced equatorial coronal hole which was centered on the solar disk on May 29, 2013, strongly suggesting a CIR origin of this storm. In fact, Zhang et al. (2007) have studied 88 major storms (Dst $\leqslant-100$ ) between 1996 and 2005 and found that while $60 \%$ were due to ICMEs, $27 \%$ to complex solar wind flows, usually due to interacting CMEs, 11 storms out of 88 were due to CIRs. The June 1, 2013 geomagnetic storm was at the strong end of CIR-induced ones. The CIR and the driving high-speed stream arrived at Wind on June 1, 2013, a forward shock preceded it by nearly one day, as can be seen in the top panel of Fig. 4. The data look very much as if the storm occurred between two high-speed streams. Careful inspection of the SDO images reveals a very faint poleward CME leaving the Sun in the questionable time, and it was not clear whether it could have caused the storm. If one looks at the Wind in situ data more carefully however, one finds an indication that the storm was triggered by a CME. The preceding fast wind is likely to have been driven by the faint polar CME from the SDO images. The second panel from the top in Fig. 4 shows magnetic field strength, which remains very steady from approximately May 28, 03:00 until $\sim$ May 31, 15:00. This is one possible interplanetary signature of ICMEs (Wimmer-Schweingruber et al.(2006)). In the same period the magnetic field turned increasingly southward, as is shown in the next panel (second from the bottom). It shows the fraction of southward field, i.e., $B_{z} / B$. Again fluctuations are seen to be small in the time preceeding the storm, i.e., likely to be part of an ICME. The field turns northward in the compression region driven by the highspeed stream from the coronal hole. Thus it looks as if the storm of June 1 may have been 


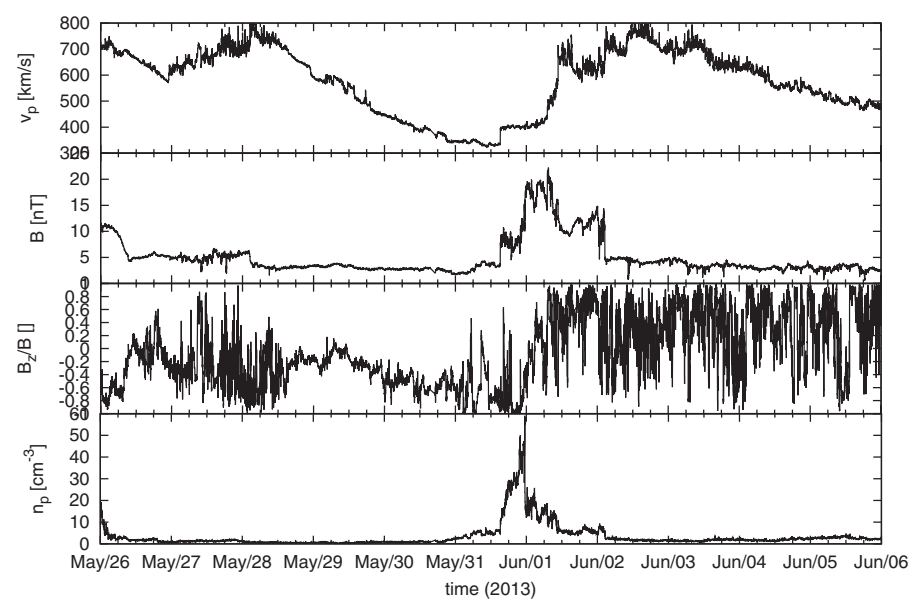

Figure 4. Solar wind proton and magnetic field data for the major geomagnetic storm as observed on June 1, 2013. Data are from the Wind SWE and MFI instrument and were acquired from the Omniweb data site (http://ftpbrowser.gsfc.nasa.gov/wind_min_merge.html)

caused by a complex interaction of a high-speed stream and its CIR interacting with the trailing edge of a preceeding ICME. Indeed if one also looks at STEREO (Kaiser et al. 2005) COR2 images, one sees clearly the polar CME which may be the driver of this storm. (Note also the large fluctuations in $B_{z} / B$ in the second high-speed stream, which are likely due to large-amplitude Alfvén waves. They too can be geoeffective.)

The message to take home from this discussion is that we still have difficulties identifying the source of a geomagnetic storm 'on the fly', especially when we have insufficient data. Having both STEREOs is very helpful in identifying Earth-directed CMEs!

\section{Where is Space Weather?}

Space weather as in "something that weathers something" is an important and ubiquitous process in the solar system and beyond. It results in spectral darkening, reddening and subdued absorption bands, which can greatly hamper the interpretation of remote sensing data of airless bodies. Sputtering by the solar wind and micrometeorite impact vaporization produce thin vapor-deposited films on regolith grains. These films contain metallic iron particles which change the optical properties of the grains (Hapke 2001). Space weather also produces the hermean and lunar exospheres (Killen and Ip 1999), and can be seen 'in action' in the sodium lines at Mercury (Killen et al. 2001). There solar wind and energetic particles can sputter the regolith, at least in the polar regions, thus liberating sodium atoms (among others) which can escape into the extended hermean exosphere. They are easily excited and their glow can then be seen (Killen et al., 2001) and the ions also measured in situ (Zurbuchen et al.2011). In fact, space weathering is likely to destroy grains in the interstellar medium and makes the interpretation of key abundances, such as that of life-enabling carbon, very difficult (Frisch \& Slavin 2003) because it condenses in grains. Thus, it is clear that space weather also matters at other locations than only Earth.

\subsection{Space Weather and (Human) Exploration}

Not only is space weather a process which slowly alters grain reflective properties and composition, but it also matters because we have an increasing number of spacecraft at other planets. For instance, the Martian Radiation Environment Experiment (MARIE) 
(Badhwar 2004), which was built to measure the radiation environment on the way to and at Mars, began malfunctioning probably due to several solar particle events and eventually ceased to operate in the fall of 2003, roughly two and a half years after launch. Incidentally, Mars Science Laboratory's (MSL) Radiation Assessment Detector (RAD) (Hassler et al.2012), by pure serendipity began measuring the radiation environment on the surface of Mars on August 7, 2012, to the day exactly 100 years after Victor Hess' discovery of cosmic rays (Hess 1912). These measurements are partially also made in preparation of human exploration of the Moon, Mars, and beyond (Olson et al.2011). Human solar system exploration will need to be accompanied by careful modeling of space weather and its effects on humans and life support systems. With increasing commercial access to space, including space tourism, this will become even more important. For instance, MarsOne plans to land humans on Mars in 2023 and 2024. In May 2013, with the application process still running until August 31, 2013, more than 78,000 people from over 120 countries had applied for selection (MarsOne 2013). Virgin Galactic, another commercial contestant for space tourism has recently added two pilots to its commercial flight team (Virgin Galactic 2013) and SpaceX, a commercial launcher company, successfully delivered cargo to the ISS (SpaceX 2013). These three examples show that commercial space utilization is increasing and larger-scale space tourism is not too distant in the future, underlining the importance of being able to predict and protect from space weather.

\subsection{An Example: Space Weather on the way to and at Mars}

Soon after MSL's launch on November 28, 2011, the RAD instrument was switched on on December 6 and started collecting data with a high duty cycle. Zeitlin et al. (2013) report the dose equivalent which would be experienced by a crew on its way to Mars and find a value of $465 \mathrm{mSv}$ for the 253-day long cruise to Mars. If this duration could be shortened to 180 days, a crew member would thus experience $2 \times 465 \mathrm{mSv} / 253 \times 180=662 \mathrm{mSv}$ on his/her way to Mars and back again (hence the factor 2). This is already uncomfortably close to NASA's career limit for astronauts, illustrating that space weather effects such as radiation are indeed a serious problem that requires careful study. The lion's share of the exposure was due to GCRs, with solar particle events only contributing approximately $5 \%$. This fraction could have been quite different and higher at other times, as it depends strongly on solar activity.

On Mars itself, the radiation environment is complex because GCRs and solar particles interact with the Martian atmosphere and soil. Because of the thin atmosphere, the production of secondary particles peaks at or close to the surface. In other words, the Pfotzer maximum (Pfotzer 1936a, b), which at Earth is in a height of approximately $20 \mathrm{~km}$, lies about at the surface of Mars. This has important consequences for possible life or biosignatures on Mars (Dartnell et al.2007, Ehresmann et al. 2011).

Space weather has other, more severe consequences at Mars. Mars lost its global, dynamo-driven magnetic field (Schubert \& Spohn 1990) a long time ago. Today it only has a remnant crustal field (Acuna et al. 1998 and 1999, Connerney et al. 2004). In fact, the field is so weak that the first probes to Mars only measured a field strength which was indistinguishable from the interplanetary field (Smith et al. 1965). Mariner 4 observed no bow shock up to its closest approach of four Mars radii. This missing magnetic field led to a gradual but massive loss of Mars' atmosphere, as recently confirmed by the Sample Analysis at Mars (SAM) instrument on MSL (Mahaffy et al. 2013). A Mars exospheric molecule, once ionized has a gyro radius which is larger than Mars' radius, and thus escapes from the planet. 


\subsection{Space Weather at Other Planets}

Space weather can also be observed at other planets or solar system bodies. We have already discussed it for Mercury, the Moon, and asteroids at the beginning of this section 3. Venus, as the next logical planet, again has no intrinsic magnetic field (Russell 1993), and subsequently, is also loosing light exospheric ions through the same process as Mars, but with the difference that the stronger interplanetary field at Venus leads to particle gyro radii which are smaller than Venus' radius.

Farther out in the solar system we encounter the gas giants Jupiter and Saturn which have strong intrinsic magnetic fields and large magnetospheres. Because they themselves can accelerate particles to high energies, they generate their own space weather. Nevertheless, solar-induced aurorae have been observed at Jupiter (e.g., Caldwell et al. 1992) and at Saturn (Trauger et al.1998) in truly beautiful Hubble images. That these planets would have aurorae was expected, as aurora-induced radio signals from Jupiter and Saturn were observed, as reviewed in Zarka (1998) and Zarka and Kurth (2005). Even farther out in the solar system the icy giants Uranus and Neptune are also exposed to space weather. Lamy et al. (2012) observed the chain of events from Sun to Uranus which lead up to the generation of aurorae at Uranus, as observed with the Hubble Space Telescope. Taking the next step, beyond the solar system, exoplanets are also expected to produce radio signals when their magnetospheres are excited sufficiently, although measurements have so far remained elusive (Bastian et al. 2000 and Lecavelier des Etangs et al.2013).

\section{Summary and Conclusions}

Disturbances are often believed to be only due to coronal mass ejections (CMEs), but we have seen that even this term (disturbances) may not be as clear cut as sometimes thought. Recurrent CIRs do disturb geomagnetism, but don't necessarily lead to the more spectacular manifestations of space weather such as aurorae or intense geomagnetic storms. What exactly disturbs space weather also depends very much on your point of view. An astronaut on his way to Mars would probably welcome an ICME as a temporary magnetic shield against the GCR, whereas the same ICME may wreck havoc in the Earth's power grid. As we just saw, space weather effects are not limited to Earth. It has important effects on the regoliths of moons, asteroids, of Mercury, and possibly even of Mars. Thus, space weather is a ubiquitous phenomenon which has been tracked from the Sun all the way out to Uranus. Of course to us it matters most at Earth as our civilization is becoming more and more dependent on miniaturized technology which is susceptible to space weather effects. The good news may be that we appear to currently be leaving the Grand Solar Maximum which has dominated the space age so far. It will be interesting to experience what the Sun has in store for us in the future.

\section{References}

Acuna, M. H., Connerney, J. E. P., Ness, N. F., Lin, R. P., Mitchell, D., Carlson, C. W., McFadden, J., Anderson, K. A., Reme, H., Mazelle, C., Vignes, D., Wasilewski, P., \& Cloutier, P., 1999. Science, 284, 790.

Acuna, M. H., Connerney, J. E. P., Wasilewski, P., Lin, R. P., Anderson, K. A., Carlson, C. W., McFadden, J., Curtis, D. W., Mitchell, D., Reme, H., Mazelle, C., Sauvaud, J. A., D'Uston, C., Cros, A., Medale, J. L., Bauer, S. J., Cloutier, P., Mayhew, M., Winterhalter, D., \& Ness, N. F., 1998. Science, 279, 1676.

Badhwar, G. D., 2004. Space Sci. Revs., 110, 131.

Barnard, L., Lockwood, M., Hapgood, M. A., Owens, M. J., Davis, C. J., \& Steinhilber, F., 2011. Geophys. Res. Lett., 38, L16103. 
Bartels, J., 1934. Terrestrial Magnetism and Atmospheric Electricity (Journal of Geophysical Research), 39, 201.

Bastian, T. S., Dulk, G. A., \& Leblanc, Y., 2000. ApJ, 545, 1058.

Bequerel, H., 1896a. Comptes rendus, 122, 420

Bequerel, H., 1896b. Comptes rendus, 122, 501

Bicknell, P. J., 1968. Isis. Journal of the History of Science Society, 59(1), 87

Birkeland, K., 1908a. The Norwegian Aurora Polaris Expedition 1902-1903, vol. 1, On the Cause of Magnetic Storms and the Origin of Terrestrial Magnetism, first section. H. Aschehoug and Co, Christiania

Birkeland, K., 1908b. Comptes rendus, 147, 539

Birkeland, K., 1911. Comptes rendus, 153, 513

Birkeland, K., 1913. The Norwegian Aurora Polaris Expedition 1902-1903, vol. 1, On the Cause of Magnetic Storms and the Origin of Terrestrial Magnetism, second section. H. Aschehoug and Co, Christiania

Bothmer, V. \& Rust, D. M., 1997. In N. Crooker, J. A. Joselyn, \& J. Feynman, eds., Coronal Mass Ejections, Geophysical Monograph Series. American Geophysical Union, Washington DC, USA, $139-146$

Caldwell, J., Turgeon, B., \& Hua, X. M., 1992. Science, 257, 1512.

Carrington, R. C., 1859. MNRAS, 20, 13

Clark, D. \& Stephenson, F., 1978. Quart. J. R. Astron. Soc., 19, 387

Connerney, J. E. P., Acuna, M. H., Ness, N. F., Spohn, T., \& Schubert, G., 2004. Space Sci. Revs., $111,1$.

Crooker, N. U. \& Horbury, T. S., 2006. Space Sci. Revs., 123, 93

Curie, P. \& Curie, M., 1898a. Comptes rendus, 127, 1215

Curie, P. \& Curie, M., 1898b. Comptes rendus, 127, 175

Dartnell, L. R., Desorgher, L., Ward, J. M., \& Coates, A. J., 2007. Biogeosciences, 4, 545

Dunlop, M. W., Taylor, M. G. G. T., Davies, J. A., Owen, C. J., Pitout, F., Fazakerley, A. N., Pu, Z., Laakso, H., Bogdanova, Y. V., Zong, Q. G., Shen, C., Nykyri, K., Lavraud, B., Milan, S. E., Phan, T. D., Rème, H., Escoubet, C. P., Carr, C. M., Cargill, P., Lockwood, M., \& Sonnerup, B., 2005. Annales Geophysicae, 23(8), 2867

Eddy, J. A., 1976. Science, 192, 1189.

Ehresman, B., Burmeister, S., Wimmer-Schweingruber, R. F., \& Reitz, G., 2011. J. Geophys. Res., 116, A10106 (1-9).

Frisch, P. C. \& Slavin, J. D., 2003. ApJ, 594, 844.

Galactic, V., 2013. Virgin galactic adds two pilots to commercial flight team. http://www . virgingalactic.com/news/item/virgin-galactic-adds-two-pilots -to-commercial-flight-team/. [Online; accessed 2013-09-10]

Galileo, G. \& Scheiner, C., 2010. On sunspots. University of Chicago Press. Translated and Introduced by Eileen Reeves and Albert Van Helden

Gilbert, W., 1600. De Magnete

Gleissberg, W., 1944. Terrestrial Magnetism and Atmospheric Electricity, 49, 243

Hapke, B., 2001. J. Geophys. Res., 106, 10039.

Hassler, D. M., Zeitlin, C., Wimmer-Schweingruber, R. F., Böttcher, S., Martin, C., Andrews, J., Böhm, E., Brinza, D. E., Bullock, M. A., Burmeister, S., Ehresmann, B., Epperly, M., Grinspoon, D., Köhler, J., Kortmann, O., Neal, K., Peterson, J., Posner, A., Rafkin, S., Seimetz, L., Smith, K. D., Tyler, Y., Weigle, G., Reitz, G., \& Cucinotta, F. A., 2012. Space Sci. Revs., 170, 503.

Hathaway, D. H., 2010. Living Reviews in Solar Physics, 7(1) URL http://www. livingreviews.org/lrsp-2010-1. Accessed 2013-09-09

Hess, V. F., 1912. Physikalische Zeitschrift, 13, 1084

Kaiser, M. L., Kucera, T. A., Davila, J. M., St. Cyr, O. C., Guhathakurta, M., Christian, E., 2008. Space Sci. Revs., 136, 5

Killen, R. M. \& Ip, W. H., 1999. Reviews of Geophysics, 37, 361

Killen, R. M., Potter, A. E., Reiff, P., Sarantos, M., Jackson, B. V., Hick, P., \& Giles, B., 2001. J. Geophys. Res., 106, 20509. 
Lamy, L., Prangé, R., Hansen, K. C., Clarke, J. T., Zarka, P., Cecconi, B., Aboudarham, J., André, N., Branduardi-Raymont, G., Gladstone, R., Barthélémy, M., Achilleos, N., Guio, P., Dougherty, M. K., Melin, H., Cowley, S. W. H., Stallard, T. S., Nichols, J. D., \& Ballester, G., 2012. Geophys. Res. Lett., 39, L07105.

Lecavelier des Etangs, A., Sirothia, S. K., Gopal-Krishna, \& Zarka, P., 2013. A\&A A, 552, A65.

Mahaffy, P. R., Webster, C. R., Atreya, S. K., Franz, H., Wong, M., Conrad, P. G., Harpold, D., Jones, J. J., Leshin, L. A., Manning, H., Owen, T., Pepin, R. O., Squyres, S., Trainer, M., \& Team, M. S., 2013. Science, 341(6143), 263

MarsOne, 2013. 78,000 sign up for one-way mission to Mars. http://www.mars-one.com/ en/mars-one-news/press-releases/11-news/437- 78000-sign-up-for-one-waymission-to-mars/. [Online; accessed 2013-09-10]

Mewaldt, R. A., Davis, A. J., Lave, K. A., Leske, R. A., Stone, E. C., Wiedenbeck, M. E., Binns, W. R., Christian, E. R., Cummings, A. C., de Nolfo, G. A., Israel, M. H., Labrador, A. W., \& von Rosenvinge, T. T., 2010. ApJ. Lett., 723, L1.

Olson, J., Craig, D., Maliga, K., Mullins, C., Hay, J., Graham, R., Graham, R., Smith, P., Johnson, S., \& Simmons, A., 2011. Voyages - charting the course for sustainable human space exploration, brochure np-2011-06-395-larc. Technical report, NASA

Pacini, d., 1912. Nuovo Cimento, Serie VI, 3, 93

Parker, E. N., 1979. Cosmical Magnetic Fields. Oxford University Press

Pfotzer, G., 1936a. Zeitschrift für Physik, 102, 23.

Pfotzer, G., 1936b. Zeitschrift für Physik, 102, 41.

Röntgen, W. C., 1895. Sitzungsberichte der Würzburger Physik.-medic. Gesellschaft, 9, 132

Russell, C. T., 1993. Reports on Progress in Physics, 56, 687.

Schubert, G. \& Spohn, T., 1990. J. Geophys. Res., 95, 14095.

Schwabe, H., 1844. Astron. Nachr., 21(495), 233.

SIDC-team, 1700-2013. Monthly Report on the International Sunspot Number, online catalogue. URL http://www.sidc.be/sunspot-data/. Accessed 2013-09-09

Smith, E. J., Davis, Jr., L., Coleman, Jr., P. J., \& Jones, D. E., 1965. Science, 149, 1241.

SpaceX, 2013. Dragon delivers cargo to station. http://www.spacex.com/. [Online; accessed 2013-09-10]

Stern, D. P., 2002. Rev. Geophys., 40, 1

Trauger, J. T., et al., 1998. J. Geophys. Res., 103, 237

Usoskin, I. G., 2013. Living Reviews in Solar Physics, 10(1) URL http://www.livingreviews . org/lrsp-2013-1 Accessed 2013-09-08

Wimmer-Schweingruber, R. F., 2006. Space Sci. Revs., 123, 471

Wimmer-Schweingruber, R. F., Crooker, N. U., Balogh, A., Bothmer, V., Forsyth, R. J., Gazis, P., Gosling, J. T., Horbury, T., Kilchenmann, A., Richardson, I., Richardson, J., Riley, P., Rodriguez, L., von Steiger, R., Wurz, P., \& Zurbuchen, T. H., 2006. Space Sci. Revs., 123, 177 .

Wittmann, A. \& Xu, Z., 1987. A\&A Suppl., 70, 83

Wolf, R., 1856. Viertelsjahrschr. Naturforsch. Ges. Zürich, 1, 151

Zarka, P., 1998. J. Geophys. Res., 103, 20159.

Zarka, P. \& Kurth, W. S., 2005. Space Sci. Revs., 116, 371.

Zeitlin, C., Hassler, D. M., Cucinotta, F. A., Ehresmann, B., Wimmer-Schweingruber, R. F., Brinza, D. E., Kang, S., Weigle, G., Bttcher, S., Bhm, E., Burmeister, S., Guo, J., Khler, J., Martin, C., Posner, A., Rafkin, S., \& Reitz, G., 2013. Science, 340(6136), 1080

Zhang, J., Richardson, I. G., Webb, D. F., Gopalswamy, N., Huttunen, E., Kasper, J. C., Nitta, N. V., Poomvises, W., Thompson, B. J., Wu, C. C., Yashiro, S., \& Zhukov, A. N., 2007. Journal of Geophysical Research (Space Physics), 112, A10102.

Zurbuchen, T. H., Raines, J. M., Slavin, J. A., Gershman, D. J., Gilbert, J. A., Gloeckler, G., Anderson, B. J., Baker, D. N., Korth, H., Krimigis, S. M., Sarantos, M., Schriver, D., McNutt, R. L., \& Solomon, S. C., 2011. Science, 333, 1862.

Zurbuchen, T. H. \& Richardson, I. G., 2006. Space Sci. Rev., 123, 31 